\title{
BOUNDARY CONDITIONS ON THE VECTOR AND SCALAR POTENTIALS IN VISCOUS THREE-DIMENSIONAL HYDRODYNAMICS*
}

\author{
By G. J. HIRASAKI (Shell Development Co., Houston, Texas) \\ AND \\ J. D. HELLUMS (Rice University)
}

1. Introduction. Solution of the Navier-Stokes equations has become possible for increasingly important and complicated problems in recent years. A considerable literature has developed on numerical methods for such problems. Most of the work has been on two-dimensional problems using the vorticity and stream function as dependent variables [1], [2]. However, some methods (Chorin [3], [4] and Harlow and Welch [5]) have been proposed in which the velocity components and the pressure are retained as dependent variables. In recent years two methods for attacking three-dimensional problems have been described (Aziz and Hellums [11], Chorin [4]). The method of Aziz and Hellums for three-dimensional problems involves formulation in terms of a vector potential. Such a formulation is possible for incompressible flows and the approach has been shown to be promising. The vector potential may be thought of as a threedimensional generalization of the usual two-dimensional formulation in terms of the stream function. A most interesting feature of this approach is that there is some difficulty in expressing the boundary conditions in the most convenient form. This difficulty is in direct contrast to the two-dimensional case where finding restrictions on the stream function on the boundary is relatively simple.

A general procedure has been developed for this boundary condition problem [6], [7] and the present work may be regarded as an extension of this prior work. In incompressible flows the velocity field may be expressed as the curl of a vector potential as in the prior work. It is also possible to express the velocity field for incompressible flows as the sum of the curl of a vector potential and the gradient of a scalar potential. This formulation in terms of two potentials is the subject of the present work. It will be shown that the use of two potentials yields much simpler boundary conditions in some cases and a general procedure will be given for finding these boundary conditions. It will also be shown that the use of two potentials makes it possible to treat a restricted class of compressible flow problems.

The basic boundary conditions are simply the restrictions on velocity of the original problem expressed in terms of the potentials and the vorticity. These basic restrictions leave a great deal of freedom. From the definitions it is clear that the vector potential is determined only up to a gradient of an arbitrary scalar and the scalar potential is determined only up to a scalar constant. A method will be given below for specifying restrictions on the potentials which are compatible with the basic boundary conditions and which lend themselves to numerical procedures.

* Received July 28, 1969. 
2. Hypothesis and basic differential equations. Iset the region of space for which we wish to determine the flow field be denoted by $V$, and let it be a regular or an infinite regular region of space as defined by Kellogg [S]. Let $S$ be the surface that is the boundary of $V$. Let the velocity field $V$ have continuous derivatives of the second order in the interior of $V$, continuous one-sided derivatives normal to $S$ on $S$, and continuous first derivatives with respect to the surface coordinates on $S$.

The development of the theory is based on classical potential theory for stationary fields. Thus, to satisfy the hypothesis it is necessary for the flow field to be stationary or for a disturbance at any point in a nonstationary field to be felt everywhere in the region instantaneously, i.e., infinite speed of sound. However, compressible, nonstationary flow may be represented if the speed of sound is sufficiently greater than the product of the maximum frequency of any disturbance and the maximum length of the region. Batchelor [9] gives a more detailed discussion of the circumstances in which the compressibility may be neglected.

The scalar potential, $\phi$, and the vector potential, $A$, are defined by the equation

$$
V=-\nabla \phi+\nabla \times A
$$

It is well known that these potentials exist under the hypothesis and that an $\boldsymbol{A}$ can be found such that

$$
\nabla \cdot A=0 \text {. }
$$

A differential equation in the scalar potential may be derived by taking the divergence of Eq. (2.1)

$$
\begin{aligned}
\nabla^{2} \phi & =-\nabla \cdot V \\
& \equiv-\Theta
\end{aligned}
$$

where $\Theta$ is the divergence of velocity or the rate of expansion of the flow field.

A differential equation in the vector potential is derived by taking the curl of (2.1) and applying (2.2):

$$
\begin{aligned}
\nabla^{2} A & \equiv \nabla(\nabla \cdot A)-\nabla \times(\nabla \times A) \\
& =-\nabla \times(\nabla \times A) \\
& =-\nabla \times V \\
& \equiv-W
\end{aligned}
$$

where $W$ is the vorticity.

The other equations required to determine the system are derived from the conservation of mass, linear momentum, and energy, together with equations of state and the constitutive equation. For simplicity assume that the fluid is Newtonian with constant coefficients of viscosity. The vorticity transport equation may be derived in the usual way by taking the curl of the equation of motion.

$$
D W / D t-W \cdot \nabla V+\Theta W=\nabla \times f / \rho+\nu \nabla^{2} W-(\nabla \rho) \times D V / D t .
$$

Boundary conditions in terms of the vorticity may be found from the specified velocity distribution using the definition of vorticity, the curl of the velocity.

3. Boundary conditions. As mentioned earlier, the specification of the velocity on 
the boundary, as is the usual basic condition in fluid mechanics, does not imply a unique set of boundary conditions on the potentials. Hence, additional restrictions must be imposed and these restrictions may be selected to simplify the procedure of solution. Of course, the additional restrictions must be compatible with the basic boundary conditions. A set of conditions which seems most advantageous is given below. These comprise one restriction on the complete boundary on the scalar potential and one on each component of the vector potential. The great advantage of the conditions given below is that it is possible to require the tangential components of the vector potential to vanish on the entire boundary.

The scalar potential is specified to have the boundary condition that

$$
\begin{aligned}
n \cdot \nabla \phi & \equiv \partial \phi / \partial n \\
& =-n \cdot V .
\end{aligned}
$$

From (2.1) this implies that

$$
n \cdot \nabla \times A=0
$$

The vector potential as defined by (2.1) and (2.2) is not unique but is arbitrary by the gradient of a harmonic function. Let

$$
A^{\prime}=A-\nabla \gamma
$$

where $\nabla^{2} \gamma=0$ is another vector potential that satisfies (2.1) and (2.2) and $\gamma$ will be specified later.

Since $n \cdot \nabla \times A=0$, the tangential projection of $A$ on $S$ may be expressed as

$$
A_{T}=\nabla_{s} \psi
$$

where

$$
\psi(P)=\int_{P_{0}}^{P} A \cdot d X .
$$

$P_{0}$ is an arbitrary point on $S$ and $\nabla_{S}$ is the surface gradient operator. Let $\gamma=\psi$ on $S$. Then

$$
\begin{aligned}
A_{T}^{\prime} & =A_{T}-\nabla_{S} \gamma \\
& =A_{T}-\nabla_{S} \psi \\
& =0 .
\end{aligned}
$$

Thus, $A^{\prime}$ is a vector potential satisfying (2.1) and (2.2) and by (3.5) has a zero tangential projection on $S$; i.e., it is normal to $S$. It is easy to show that the vector potential $A^{\prime}$ with these properties is unique [7]. Hence, for boundary conditions on the tangential components of the vector potential one may require that the components vanish. Such a restriction is not possible on the normal component. Instead, from the requirement that $A$ be solenoidal, we have

$$
\frac{\partial}{\partial x^{3}}\left[h_{1} h_{2} A(3)\right]=0
$$

if $\left(x^{1}, x^{2}, x^{3}\right)$ is an orthogonal coordinate system with $x^{3}$ in the normal direction. In the special case of a flat boundary with Cartesian coordinates (3.6) reduces to $\partial A(n) / \partial n=0$. 
On the scalar potential we have the differential equation (2.3) with the condition given by Eq. (3.1). From these equations we see that $\phi$ is an arbitrary constant for the important special case of incompressible flows bounded by solid surfaces. In the general case it is clear that $\Theta$ is nonzero and time-dependent and the scalar potential may be a function of time.

On the vector potential we have (now letting $\boldsymbol{A}$ denote what previously was called either $\boldsymbol{A}$ or $\boldsymbol{A}^{\prime}$ ) the vector differential equation (2.4) with conditions that the tangential components of $A$ vanish, as by Eq. (3.5). The condition on the normal component is given by (3.6).

It can easily be proved that the vector potential that is a solution of (2.4) with boundary conditions (3.5) and (3.6) does in fact satisfy the condition $\nabla \cdot A=0$. The proof is similar to that given by Sommerfeld [10] for the case of an unbounded flow field.

The equations and conditions are written above formally as if $\Theta$ and $W$ were specified in which case (2.3) and (2.4) could be solved independently to yield the potentials. However, the equations are coupled into the system of equations as described previously. It has been found to be advantageous in numerical work to solve (2.3) and (2.4) at each time step as if uncoupled. Then the coupling and other nonlinearities are taken into account by an iterative procedure. In this way it is possible to make use of some of the well-developed linear methods of numerical analysis.

Aziz and Hellums [11] give a numerical solution for three-dimensional time-dependent free convection using the approach discussed here. They considered the special incompressible case in which the scalar potential plays no role.

4. Conclusion. The theory presented here gives a general method for determining the simplest boundary conditions on the scalar and vector potentials consistent with a specified velocity distribution on the boundary. In incompressible flows, the scalar potential can always be required to vanish identically. However, imposing this requirement may cause the boundary conditions on the vector potential to be more complicated and more difficult to determine. Hence, it is advantageous to use both potentials except in cases where there is no flux across the boundaries.

\section{REFERENCES}

[1] J. E. Fromm, Report LA-3522, Los Alamos Scientific Laboratory, 1067

[2] K. E. Torrance, J. Res. Nat. Bur. Standards 72B, No. 4 (1968)

[3] A. J. Chorin, A. E. C. Research and Development Report No. NYO-1480-61, New York, 1966

[4] —_, Bull. Amer. Math. Soc. 73, 928 (1967)

[5] F. II. Harlow and J. E. Welch, Phys. Fluids 10, 927 (1967)

[6] G. J. Hirasaki and J. D. Hellums, A general formulation of the boundary conditions on the vector potential in three-dimensional hydrodynamics, Quart. Appl. Math. 26, 331 (1968)

[7] G. J. Hirasaki, A general formulation of the boundary conditions on the vector potential in three-dimensional hydrodynamics, Ph.D. thesis, Rice Univ., 1967

[8] O. D. Kellogg, Foundations of potential theory, Dover, New York, 1953

[9] G. K. Batchelor, An introduction to fluid dynamics, Cambridge Univ. Press, New York, 1967, pp. $164-171$

[10] A. Sommerfeld, "Mechanics of deformable bodies," Lectures on theoretical physics, II, Academic Press New York, 1950 , p. 150

[11] K. Aziz and J. D. Hellums, Numerical solution of the three-dimensional equations of motion for laminar natural convection, Phys. of Fluids 10, 314 (1967) 